\title{
Relation Between the Absorption Spectra and the Chem- ical Constitution of Dyes: XXIX. Interaction of Direct Azo Dyes in Aqueous Solution
}

\author{
May N. Inscoe, John H. Gould, Mary E. Corning, and Wallace R. Brode
}

\begin{abstract}
Interaction of dyes in aqueous solution results in differences in the spectrum of the mixture when compared with the sum of the spectra of the individual dyes. A general survey, chiefly with azo dyes, was made to determine the effect of dye structure on this interaction.

The effect of other factors on the interaction of direct azo dyes was investigated. The addition of alcohol or non-ionic detergent prevents this interaction, while the presence of inorganic salts or an increase in dye concentration appears to increase the extent of interaction. The effect of acids and bases is dependent upon the nature of the dyes in the mixture.

The results obtained are consistent with the assumption that the forces involved in the interaction of direct azo dyes in mixtures are the same as those causing the aggregation of individual dyes and those binding these dyes to fibers.
\end{abstract}

\section{Introduction}

Dyes in aqueous solution often show a tendency toward association, and it is probable that the forces involved are closely related to those responsible for binding the dyes to fibers. This association may be between similar molecules or ions in solutions of individual dyes or between unlike molecules or ions in dye mixtures. In this paper the term. "aggregation" is used to refer to the association of like molecules, while the association of unlike dye molecules in mixtures is designated as "interaction."

The association of like dye molecules or ions in aqueous solutions to form dimers or higher aggregates has been studied intensively and is so common a phenomenon that it has been considered a general property of dyes, closely related to the property of color [1]. ${ }^{2}$ Those cases in which aggregation is not observed may be regarded as exceptions in which steric [2] or electrical [3] influences prevent the close association of the molecules.

Striking changes in the absorption spectra of many basic dyes are produced by dimerization and further aggregation, and such association has been studied spectrophotometrically. In the case of azo dyes, changes in absorption spectra arising from aggregation are not as marked, and other physical measurements have more commonly been used to demonstrate the aggregation [4].

The nature of the forces involved in this aggregation of dyes is not yet clear. Some proposed explanations include hydrogen bonding, van der Waal's forces, the interaction of $\pi$-electrons, or the coupling of electron oscillators. It is very probable that more than one factor is operative in most cases.

The association of unlike ions or molecules in mixtures of dyes has not been as fully investigated as that of individual dyes, although the absorption spectra of dye mixtures in many cases suggest that such interaction occurs. Scheibe observed such

\footnotetext{
1 This research was supported by the United States Air Force through the Office of Scientifle Research of the Air Research and Development Command. 2 Figures in brackets indicate the literature references at the end of this paper.
}

behavior in mixtures of cyanine dyes [5], while Rabinowitch and Epstein found indications that mixed double ions were present in mixtures of thionine and methylene blue in water [1].

This interaction of dyes in mixtures has been most widely observed with direct cotton dyes, and the spectra of mixtures of substantive azo dyes in aqueous solution often differ from the spectra that would be predicted by the addition of the spectra of the individual components. The results obtained from the study of several such mixtures $[6,7]$ show that interaction to form a mixed dye complex often takes place, with equilibrium between the dyes and the complex, and suggest that the forces involved in this interaction are related to those operating in the dyeing of cotton.

In view of the apparent close relationship between the aggregation of dyes, the interaction of dyes in mixtures, and the binding of dyes to fibers, a wider survey of the interaction of azo dyes and the factors influencing this interaction was felt to be desirable. The results of this survey are described below.

\section{Experimental Procedure}

\subsection{Dyes Used}

The majority of the dyes used were commercial samples. When greater purity was desired, conventional methods for purification were employed. Some of the dyes had been prepared for other investigations by standard methods of synthesis and purification.

Stock solutions of dyes were prepared by dissolving weighed samples in distilled water and diluting to volume. In use, suitable aliquots of these stock solutions were diluted in volumetric flasks. For most measurements the final concentration used was chosen to give a maximum absorbance of the principal long-wavelength band between 0.5 and 1 when measured in a $2-\mathrm{cm}$ cell; these concentrations ranged from 5 to $20 \mathrm{mg}$ /liter. 
The names used in this paper for the commercial dyes are those given by the Technical Manual and Year Book of the American Association of Textile Chemists and Colorists, Vol. XXXII, 1956, as representing the best-known prototype of the dye.
The dyes included in this survey are shown in table 1, where each dye has been assigned an arbitrary number for convenience of reference in this paper.

TABLE 1. Dyes included in the survey

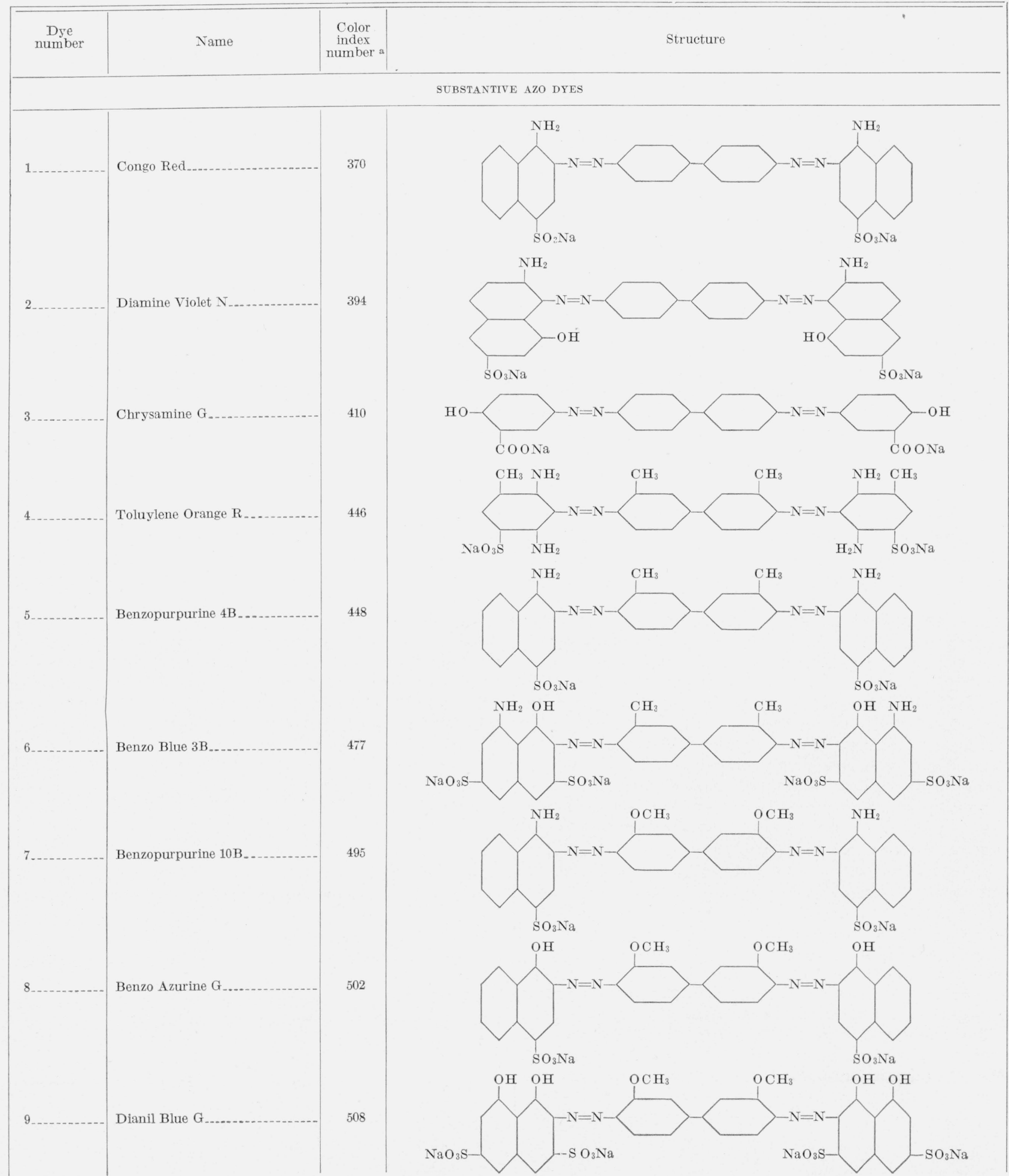


Table 1. Dyes included in the survey-Continued

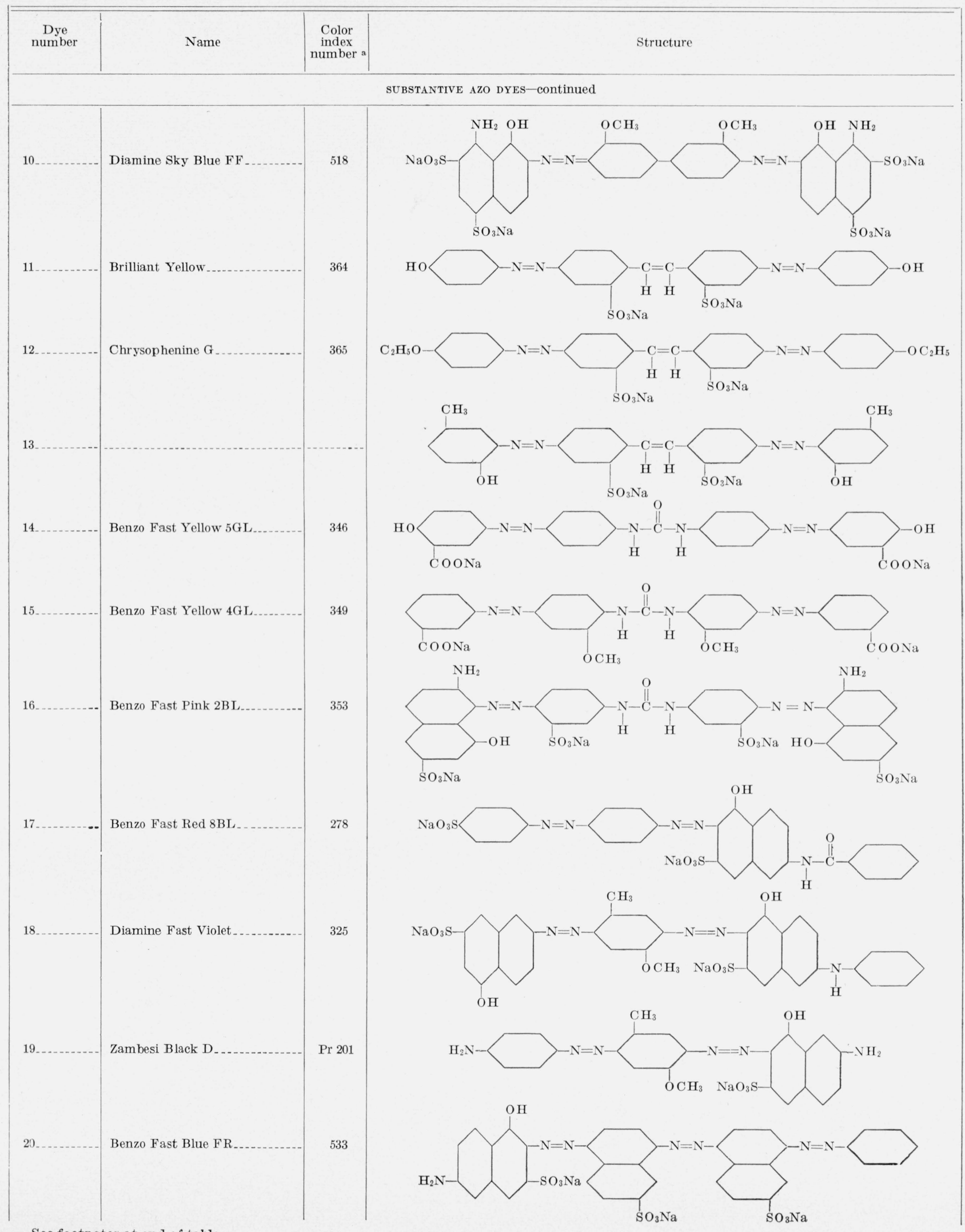

See footnotes at end of table. 
Table 1. Dyes included in the survey - Continued

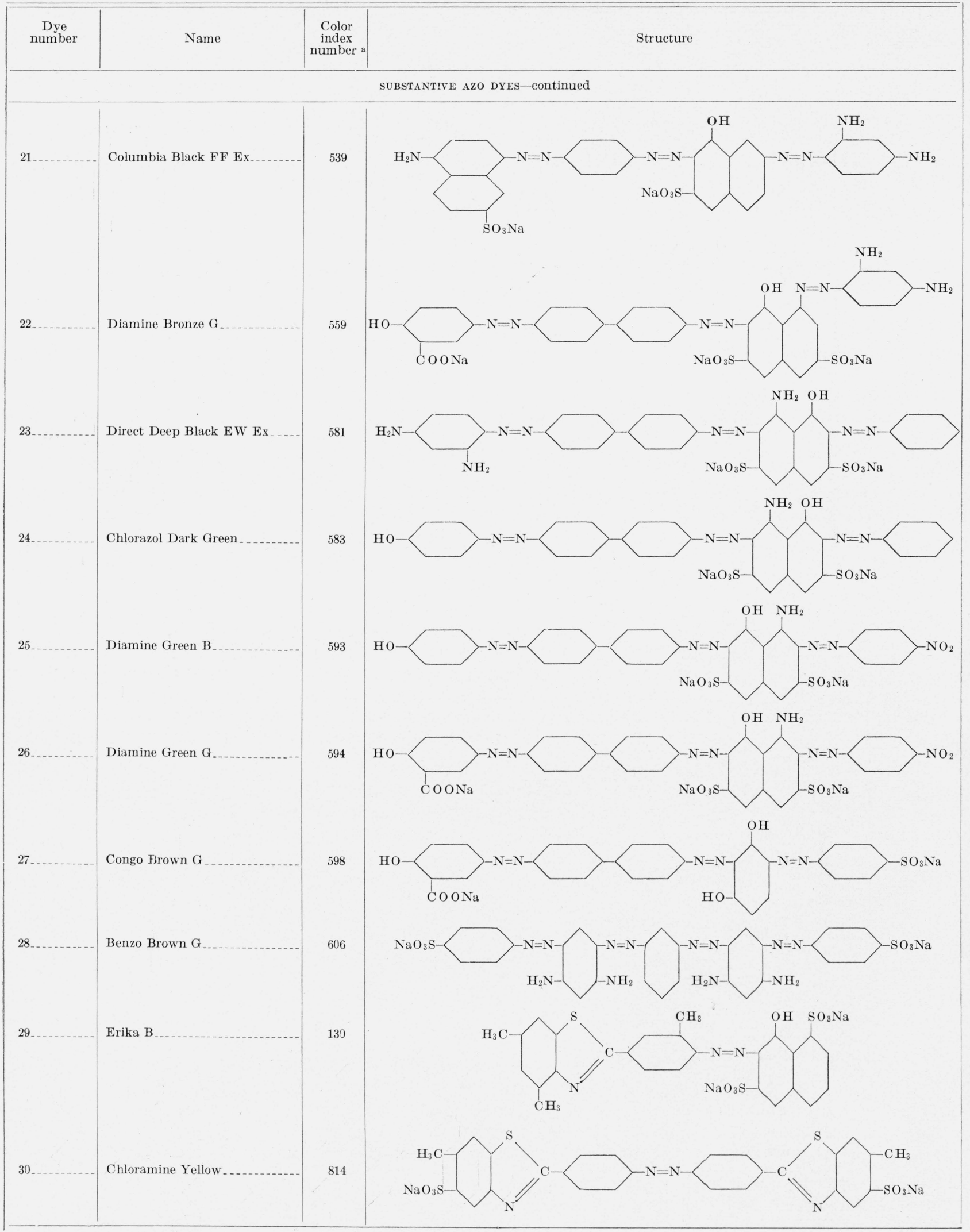

See footnotes at end of table. 
TABLE 1. Dyes included in the survey Continued

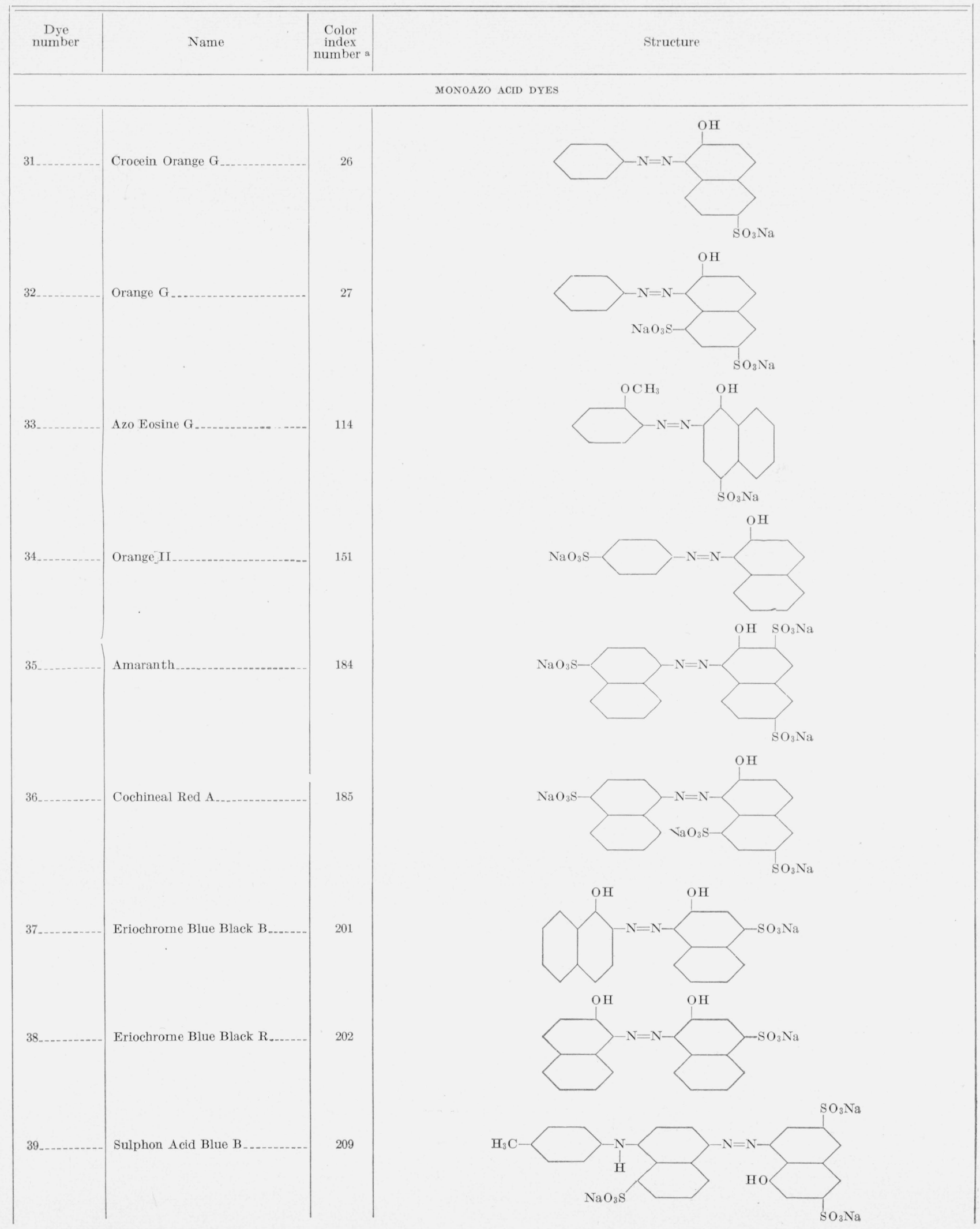

See footnotes at end of table. 
TABLE 1. Dyes included in the survey - Continued

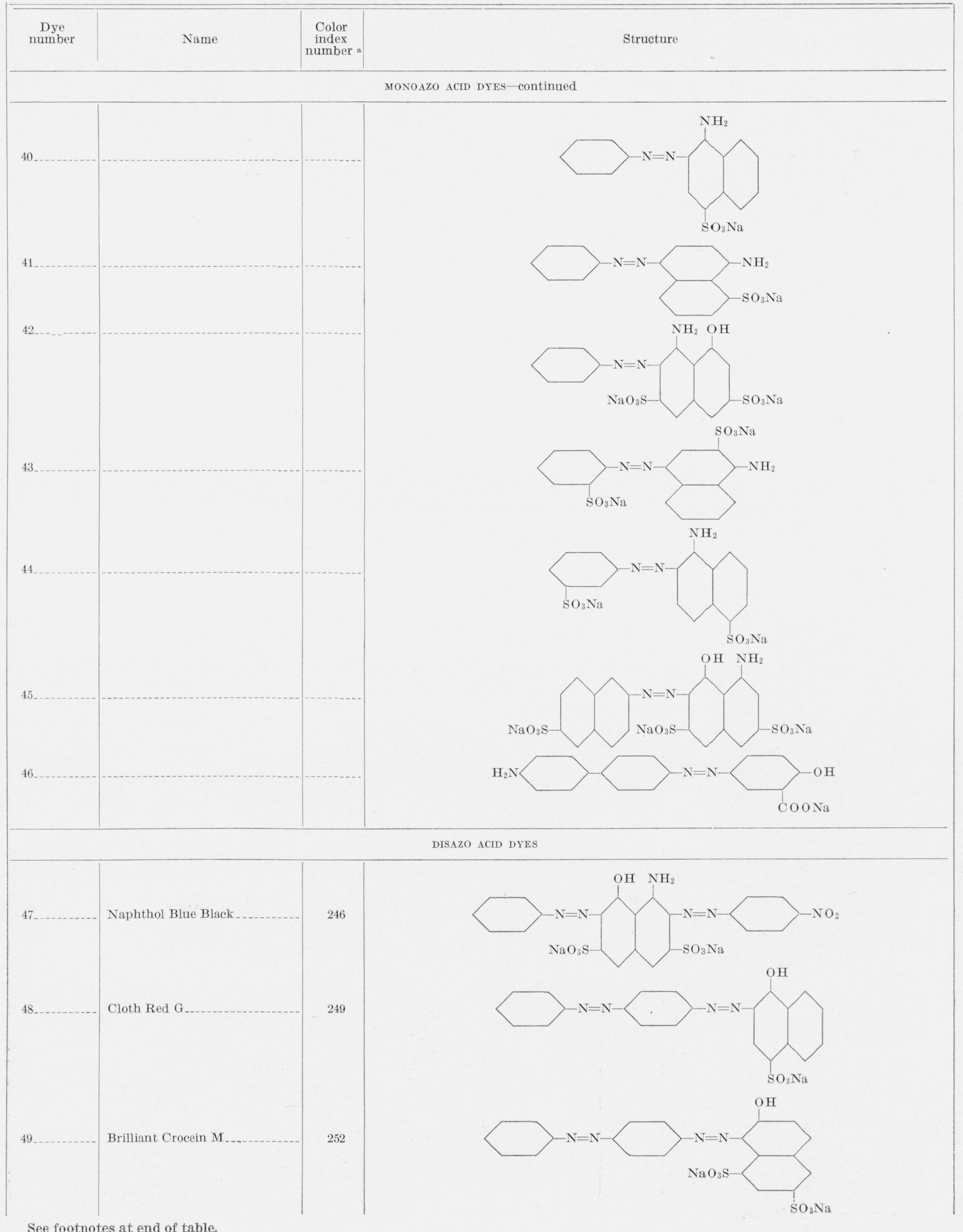


TABLE 1. Dyes included in the survey-Continued

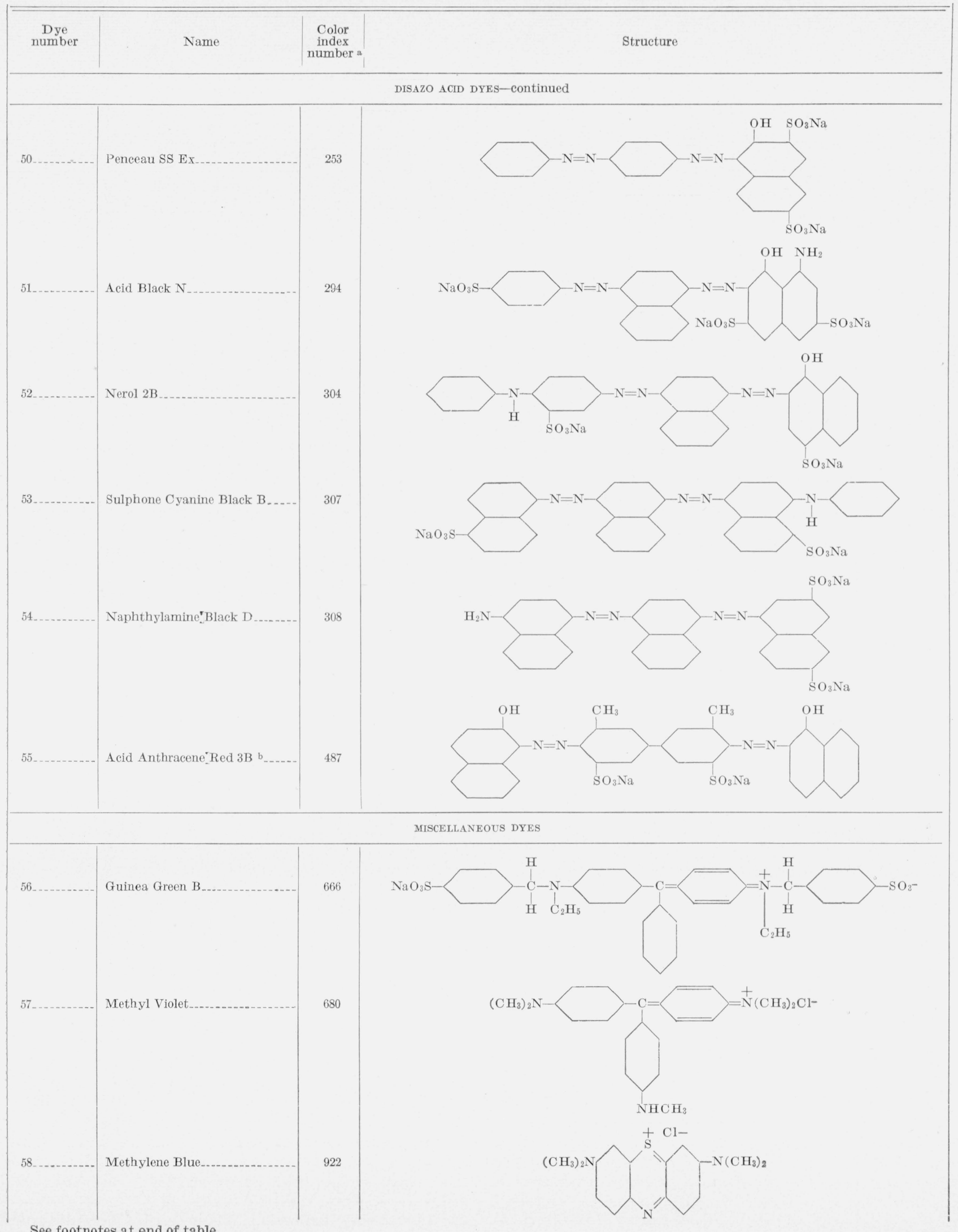

See footnotes at end of table. 
TABLE 1. Dyes included in the survey-Continued

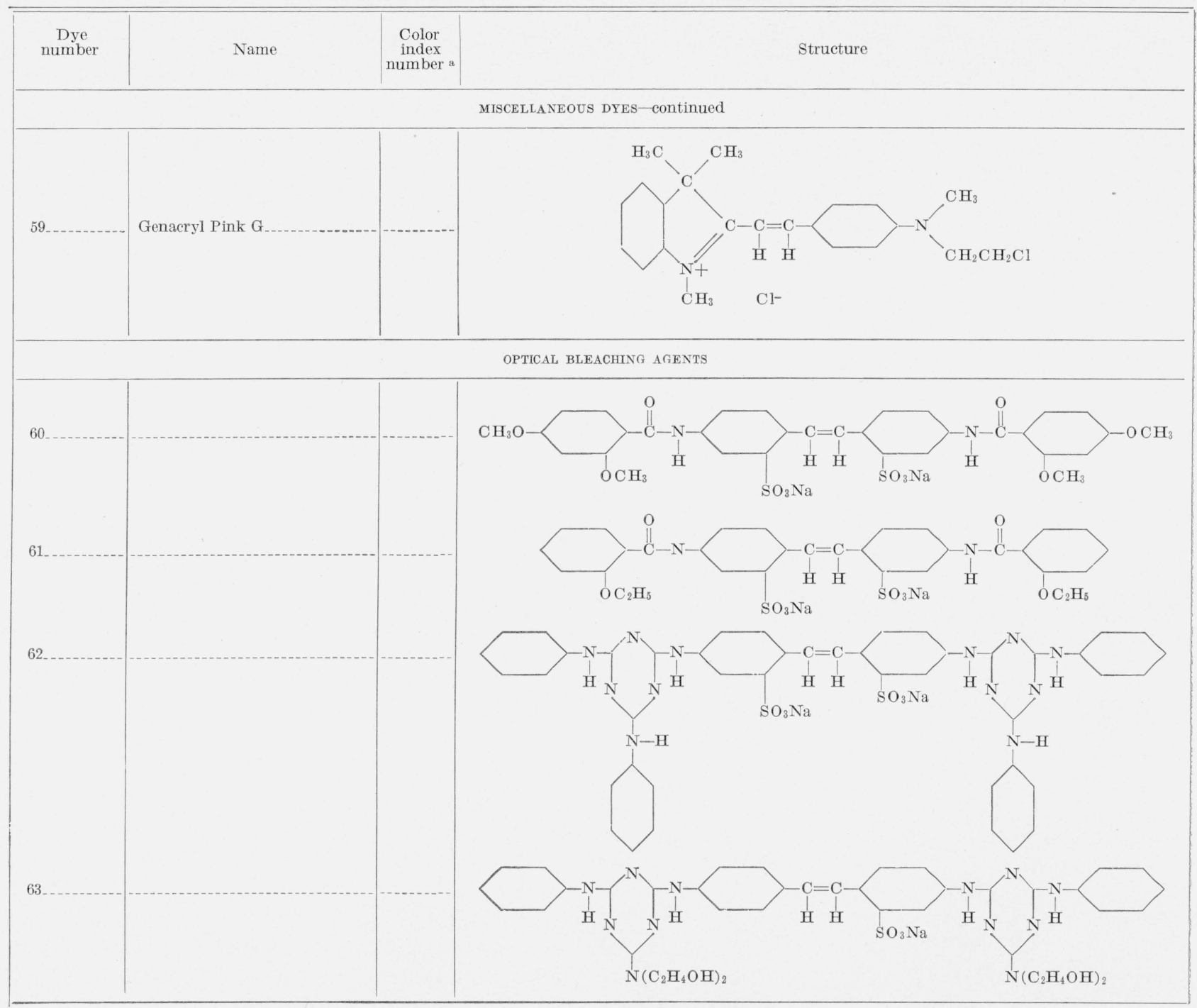

a From Rowe, Colour Index, 1st ed., Society of Dyers and Colourists, Bradford, England, 1924.

b According to Ventkataraman (Synthetic Dyes, p. 519) it has been stated recently that the sulfonic acid groups are ortho to the biphenyl linkage.

\subsection{Measurement of Absorption Spectra}

The absorption spectra were determined by means of a Cary Recording Spectrophotometer (model 12) with matched fused quartz absorption cells, and with the solvent as the reference standard.

\section{3. "Sum" and "Mixture" Curves}

The existence of association effects may be demonstrated through changes in the absorption spectra of mixtures as compared with the summation of the separate curves of the two components. The "sum" curve can be obtained by the simple addition of the curves of each dye determined separately, but with the availability of a high-speed recording spectrophotometer, it is more easily produced by placing the dye solutions in two cells arranged one behind the other. To obtain the "mixture" curve, one of these cells was filled with solvent while the other contained a mixture of the two dyes, each at the same concentration as in the separate dye solutions. For purposes of comparison the spectrum of each individual dye was also determined, so that in all, four curves have been recorded for each pair of dyes studied.

When the sum and mixture curves were identical (that is, the spectra were additive) it was considered that there was no association (see fig. 1); while differences between the two curves (nonadditive spectra) were taken as evidence of interaction. Typical association effects are shown in figures 2 and 3.

Effects other than association, such as aging, precipitation, changes in $p \mathrm{H}$, or phototropic changes, may also cause differences between the sum and mixture spectra. When aging, plating out, or precipitation was suspected, better results were obtained 


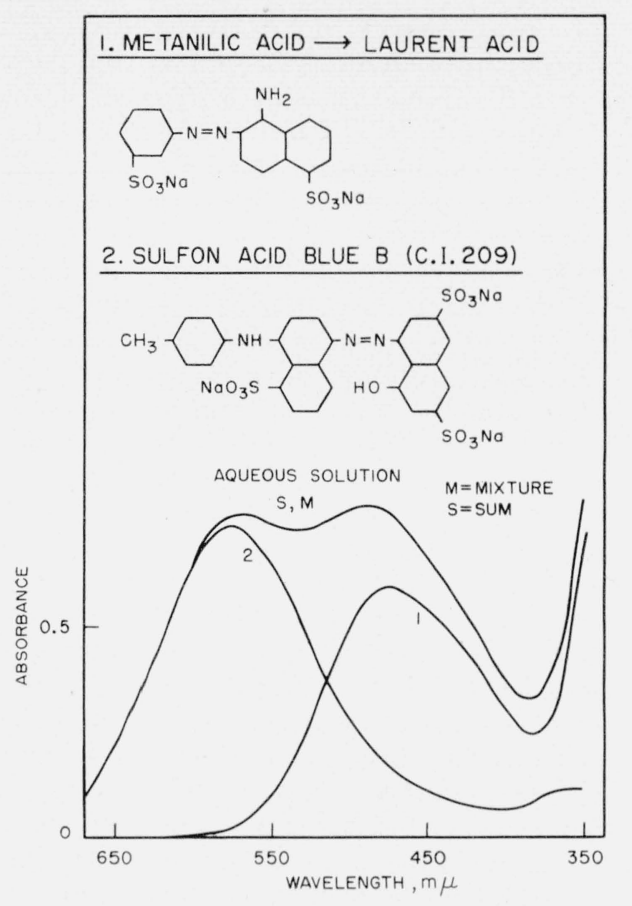

Figure 1. Sum and mixture spectra for dye No. 44 (15 $\mathrm{mg} /$ liter) with Sulfon Acid Blue B (10 mg/liter).

Length of cells equals $2 \mathrm{~cm}$.

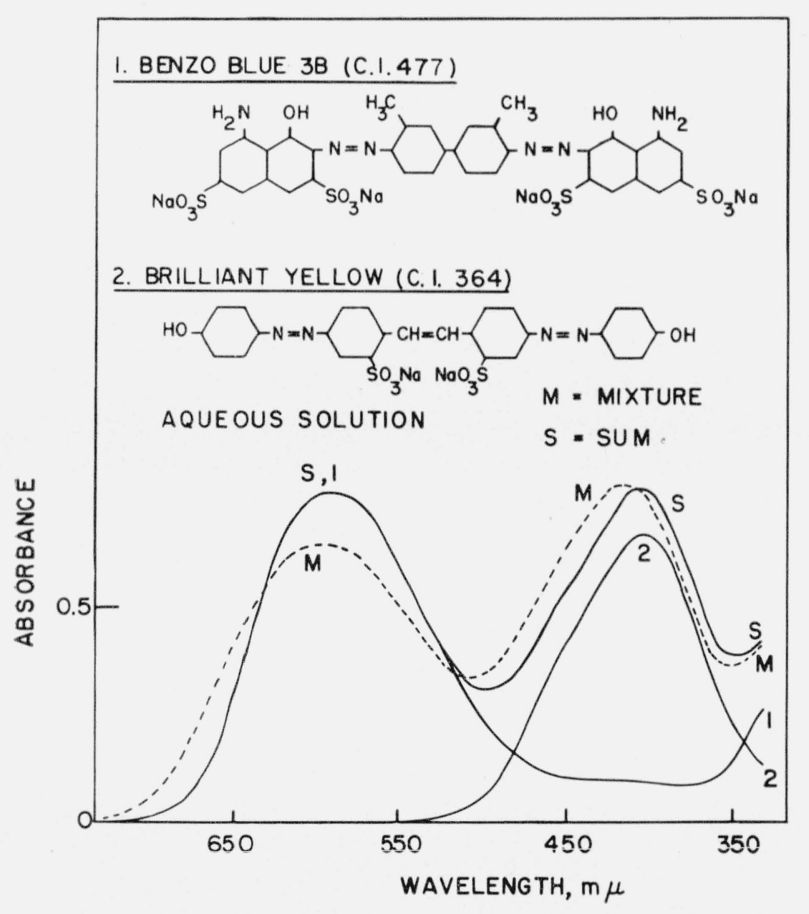

Figure 2. Sum and mixture spectra for Benzo Blue $3 B$ (10 $\mathrm{mg} /$ liter) with Brilliant Yellow (5 $\mathrm{mg} /$ liter). Length of cells equals $2 \mathrm{~cm}$.

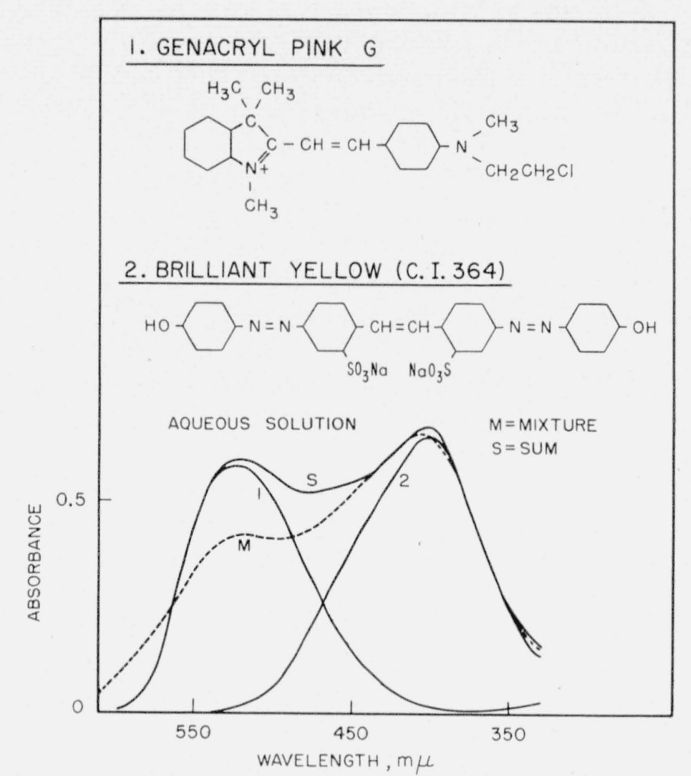

Figure 3. Sum and mixture spectra for Genacryl Pink $G$ (10 mg/liter) with Brilliant Yellow (5 $\mathrm{mg} /$ liter).

Length of cells equals $2 \mathrm{~cm}$.

by preparing the solutions immediately before measuring. For dyes sensitive to variations in $p \mathrm{H}$, caused by dissolved carbon dioxide or by the presence of a second dye in the mixture, control by a buffer was necessary. Phototropic dyes were allowed to stand in controlled light conditions until equilibrium was established.

\subsection{Irradiation of Solutions}

In the study of the phototropism of dye mixtures, the solutions were irradiated during the measurement of the spectra in the manner described in an earlier paper [8].

\section{Results}

\subsection{A General Survey of the Interaction of Dyes in Solution}

The association of dyes in mixtures, as shown by the sum and mixture curves, is described for various groups of dyes.

\section{a. Azo Dyes Substantive for Cotton}

Thirty substantive azo dyes, representing different structural types, were studied in various combinations. In general, the results obtained with these dyes confirm the observations of earlier workers $[6,7]$. Mixtures of direct dyes usually give nonadditive spectra. As a rule, where the absorption bands are sufficiently well resolved, the absorption band occurring in the visible region and associated with the dye absorbing at shorter wavelengths increases in intensity, while that associated with the dye absorbing at longer wavelengths decreases. At the 
same time, both absorption bands show a bathochromic shift. In cases where the bands overlap badly, the spectral changes cannot be classified as well, but they are still observable.

Disazo dyes derived from benzidine, o-dianisidine, or $o$-tolidine (Nos. 1 to 10$)^{3}$ appear to be most active in such dye interactions. Nonadditive spectra are observed with mixtures in which both dyes are of this type as well as with mixtures in which the second component is a direct azo dye of another type. Such dyes include disazo stilbene or urea derivatives, trisazo dyes, or disazo dyes of the type $\mathrm{A} \rightarrow \mathrm{M} \rightarrow \mathrm{J}$-acid (or $\gamma$-acid) in which the substantivity is thought to be due to the presence of $J$-acid or $\gamma$-acid [9].

The changes in spectrum that are observed when one component of the mixture consists of a benzidinetype dye containing a naphthionic acid substituent (as for example Benzopurpurine 10B, No. 7) are often much less marked than the changes observed with benzidine dyes containing other groups. The substitution of NW-acid for the naphthionic acid groups of the Benzopurpurine 10B to produce Benzo Azurine G (No.8) appears to increase the activity of the dye. It is possible that the amino group of the former is less favorable to dye interaction than the hydroxyl group.

Disazo derivatives of $4,4^{\prime}$-diaminostilbene- $2,2^{\prime}$ disulfonic acid (Nos. 11 to 13 ) or $4,4^{\prime}$-diamino-N, N' $^{\prime}$ diphenylurea (Nos. 14 to 16) show a marked interaction with benzidine dyes, but the spectral differences observed with these dyes when the second component of the mixture is a J-acid or $\gamma$-acid derivative are much less pronounced. The stilbene dyes in particular show only slight interaction with dyes of this latter type.

The differences in sum and mixture curves obtained with mixtures in which one component is a trisazo dye (Nos. 20 to 27) are usually not as great as those observed when both components are disazo dyes.

Of the direct dyes considered, the type showing the least apparent interaction in most mixtures was the unsymmetrical type, $\mathrm{A} \rightarrow \mathrm{M} \rightarrow \mathrm{J}$-acid (or $\gamma$-acid),

${ }_{3}$ The dye numbers refer to the dyes in table 1 . where $\mathrm{M}$ is a phenyl derivative (Nos. 17 to 19).

Two direct monoazo dyes containing the benzothiazole group (Nos. 29 and 30) were investigated briefly. They behave in much the same manner as other direct dyes, although these dyes do not appear to have as great a tendency for interaction as do the benzidine dyes.

There are two general situations in which interaction of direct azo dyes might be expected to occur but where none is apparent from the spectra. One is the situation in which both components of a mixture have similar or identical terminal groups. Although each of these components may give nonadditive spectra with most other direct azo dyes of various types, the spectrum of the mixture is additive when the terminal groups are identical, or very nearly additive when the terminal groups are similar (see table 2). The additive spectra observed in these cases cannot be attributed to the proximity of the absorption bands of the dyes, for nonadditive spectra have often been observed with pairs of dyes whose absorption bands are even less widely separated. Nor can these additive spectra be taken as a positive indication that no interaction has occurred. Aggregation of some individual azo dyes with increasing dye concentration is known to take place, but this aggregation does not necessarily produce marked spectral anomalies [2], particularly at the concentrations used in these studies. Therefore, if the forces involved in the interaction of dyes in mixtures are related to those causing the aggregation of an individual dye, as well may be the case, it is possible that the interaction of two dyes possessing similar terminal groups may also occur without producing marked changes in the spectrum.

The second general situation in which additive spectra are obtained from mixtures of direct azo dyes is that in which both components of the mixture bear sulfonic acid groups on the central portion of the molecule (see table 2).

Chrysophenine $\mathrm{G}$ is a dye that is not appreciably aggregated in aqueous solutions, either by increased concentration or by added salts [10]. This has been explained as resulting from the mutual repulsion of

TABLE 2. Mixtures of direct dyes giving additive spectra

\begin{tabular}{|c|c|c|}
\hline Components of mixture & Groupings invoived \\
\hline Congo Red (No. 1) and Benzopurpurine $4 \mathrm{~B}(\mathrm{No} .5)_{\ldots} \ldots$ &
\end{tabular}


TABLE 2. Mixtures of direct dyes giving additive spectra-Continued

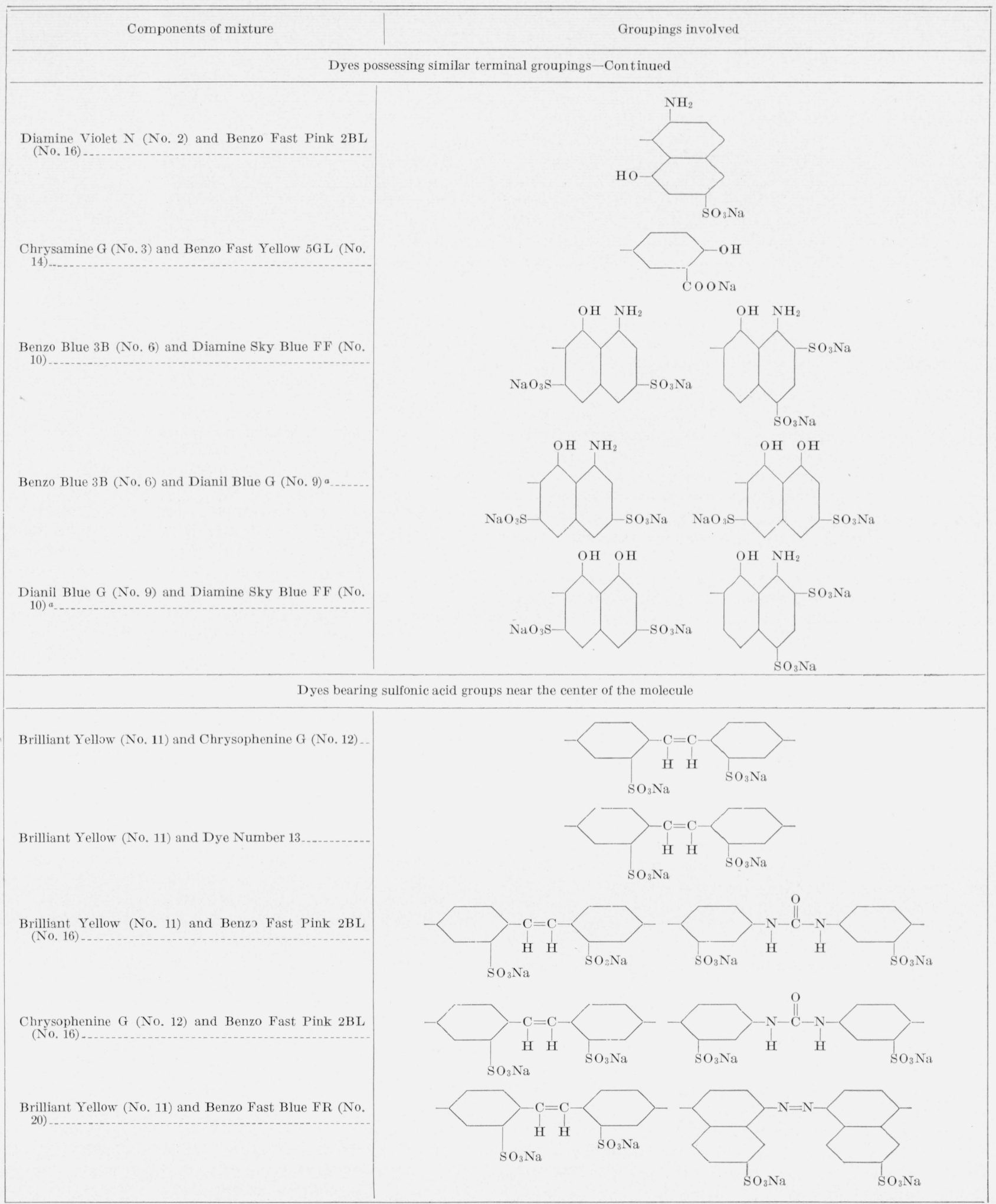

a The spectra of these mixtures are not completely additive but are very nearly so. 
the sulfonic acid groups [11]. A steric effect may also be involved, with the bulky sulfonic acid groups interfering with the close approach of the dye molecules, thus preventing aggregation. The apparent lack of interaction in mixtures of dyes possessing sulfonic acid groups near the center of the molecule may very well arise from the same causes, for the electrical repulsion or the bulkiness of the sulfonic acid groups would be expected to interfere with the interaction of such dyes as well as with their aggregation. The slight apparent interaction observed in buffered mixtures of Chrysophenine G and Benzo Fast Pink 2BL suggests that the spacing of the sulfonic acid groups are sufficiently different in these two dyes to permit a slight interaction when the interference of the sulfonic acid groups is counteracted by the presence of salts. Inorganic salts appear to enhance interaction in the same way that they tend to increase the degree of aggregation of individual dyes.

\section{b. Acid Dyes}

Acid dyes in solution tend to be molecularly dispersed, although in some cases increasing salt concentration may bring about dimerization or slight aggregation [12]. It is therefore to be expected that such dyes also show little tendency for interaction with other dyes. Many mixtures containing acid dyes give additive spectra under all conditions, while in several other cases the spectra are additive unless the solutions contain added inorganic salts. In all cases where nonadditive spectra are observed with acid dyes, the spectral differences are not great.

Most of the 16 monoazo acid dyes studied (Nos. 31 to 46 ) show no interaction with each other, and the spectra obtained from mixtures of these monoazo dyes are generally additive.

One exception to this generalization is the dye Sulphon Acid Blue B (No. 39). Although this dye gives additive spectra with many monoazo dyes (see fig. 1), it gives nonadditive spectra with several 1-arylazo-2-naphthol derivatives. The presence of a sulfonic acid group in the 8 position of the naphthol portion apparently prevents this interaction. Since Sulphon Acid Blue B is also anomalous in its behavior with direct dyes, it seems that the nonadditive spectra observed with this dye are not related to the interactions being considered in this survey.

Several monoazo acid dyes give slightly nonadditive spectra with direct disazo dyes, particularly in buffered solutions, which increase the salt concentration. No interaction is observed when the disazo dye bears sulfonic acid groups near the center of the dye molecule or when the monoazo dye has sulfonic acid groups on both aryl nuclei. Additive spectra are also obtained in cases where both components contain similar groups; e. g., a mixture of Azo Eosine G (No. 33) and Benzo Azurine G (No. 8) gives an additive spectrum. The observation by Neale and Stringfellow [6] that the spectrum obtained from a mixture of Sky Blue FF and $o$-anisidine $\rightarrow$ Chicago acid is nonadditive is unexpected in the light of our observations. Monoazo dyes containing amino groups show less tendency toward interaction than do similar dyes containing hydroxyl groups, this being another case in which amino groups appear to be less active than hydroxyl groups in promoting interaction.

Monoazo dyes give no apparent interaction with trisazo dyes; and the interaction with dyes which owe their substantivity to the presence of J-acid groups is usually very slight.

These generalizations for the monoazo acid dyes also hold true for the nine disazo acid dyes investigated (Nos. 47 to 55). In those cases where nonadditive spectra are obtained with these dyes the spectral differences are usually more pronounced than those observed with the monoazo dyes, although they are still slight when compared to the differences observed with most direct dyes. This apparent greater tendency for interaction of disazo acid dyes as compared with monoazo acid dyes is to be expected in view of the increased molecular weights and the presence of more aromatic nuclei in the disazo acid dyes.

\section{c. Miscellaneous Dyes}

The study of the interaction of dyes in aqueous solution was extended briefly to other types of dyes.

Three basic dyes were considered-Methyl Violet (No. 57), Methylene Blue (No. 58), and Genacryl Pink G (No. 59). Mixtures of these dyes with each other give nonadditive spectra, but in acetate buffers $(p \mathrm{H} 4.7$ and 5.7) the spectra of the mixtures are additive. Michaelis [3] has observed that various basic dyes that give evidence of aggregation in neutral solutions are not aggregated in solutions sufficiently acid to cause the dyes to exist as doubly charged cations. The $p \mathrm{H}$ of the acetate buffers used here is not sufficiently low to cause this change, and the lack of interaction in the buffered solutions cannot be explained by the presence of excess positive charges on the dye molecules.

The spectra of mixtures of these basic dyes with representative direct azo dyes give marked evidence of interaction, both with and without an acetate buffer (see figs. 3 and 4). The differences in the sum and mixture spectra are usually much greater in the absorption band associated with the basic dye than the region where the azo dye absorbs. In several cases the resulting changes in absorption are accompanied by hypsochromic shifts rather than the bathochromic shifts generally observed with mixtures containing only azo dyes. In the case of Methylene Blue, the interaction of the dyes is accompanied by the reduction of Methylene Blue by the azo dye.

The interaction of basic dyes with azo dyes is not, limited to substantive dyes, since the acid dya aniline $\stackrel{\text { acid }}{\longrightarrow} \mathrm{H}$-acid (No. 42) also gives a nonadditive spectrum with Methyl Violet. In such cases the interaction of the dyes may be strengthened by the electrical attraction between the oppositely charged dye ions. A somewhat similar effect is observed when direct dyes on cotton are "topped" by small amounts of basic dyes [13]. The direct dye appears to act as a mordant for the basic dye, which would not otherwise adhere to the cotton. 


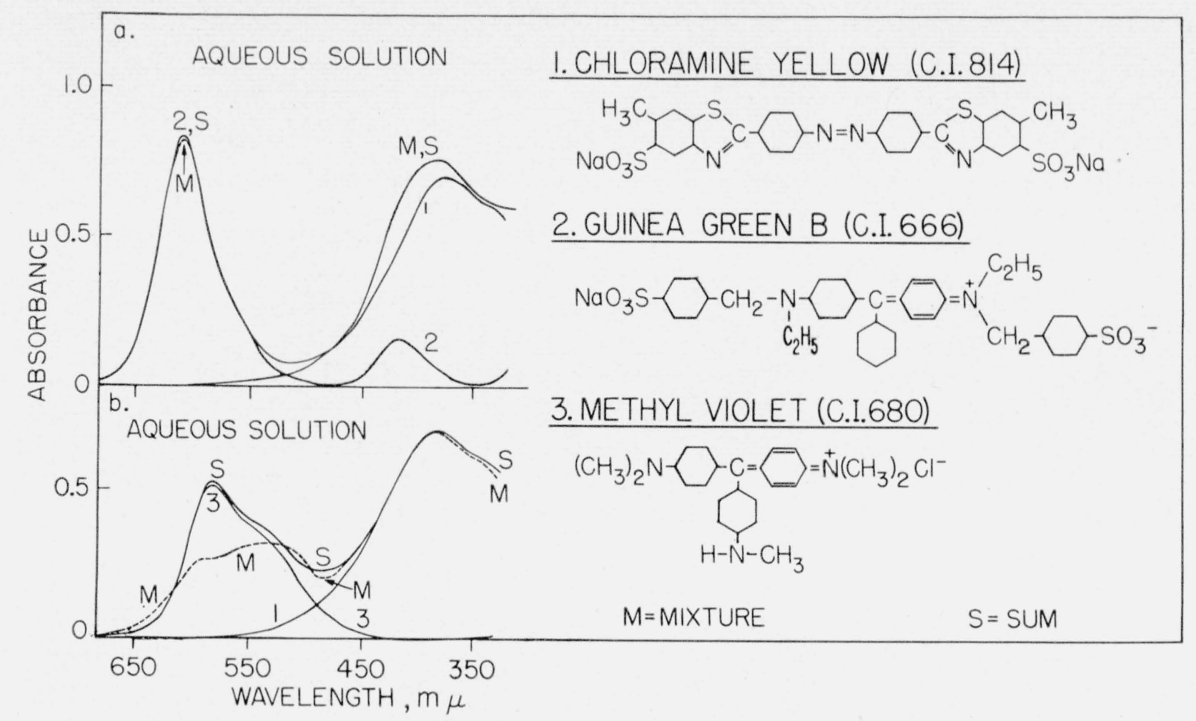

Figure 4. Sum and mixture spectra for Chloramine Yellow (10 mg/liter) with (a) Guinea Green B (5 mg/liter) and (b) with Methyl Violet (2 $\mathrm{mg} /$ liter).

Length of cells equals $2 \mathrm{~cm}$.

The acid triphenylmethane dye, Guinea Green B (No. 56), containing only two amine groups, gives additive spectra with azo dyes (fig. 4a) and with Genacryl Pink G, while with Methylene Blue the spectrum is almost additive. With Methyl Violet, however, there is evidence of some interaction either with or without the use of an acetate buffer. Sheppard and Geddes, in discussing structural effects on the aggregation of dyes, mention that increasing concentration has little effect on the spectra of the diamino triphenylmethane dyes, indicating that these dyes have little or no tendency for aggregation, although the reason for this is not apparent [2]. The tendency of Guinea Green B to give additive spectra with most other dyes may well arise from the same cause.

\section{d. Optical Bleaching Agents}

These compounds are colorless fluorescent substances absorbing in the near ultraviolet and possessing substantivity for cotton. Many of them are aryl amido or substituted amino derivatives of $4,4^{\prime}$. diaminostilbene-2,2'-disulfonic acid [14]. Mixtures of four of these compounds (Nos. 60 to 63 ) with several substantive benzidine dyes give nonadditive spectra (see fig. 5b). The spectral changes observed in these mixtures are very similar to those observed in mixtures of two direct azo dyes, except that the absorption bands of both components usually decrease in intensity, whereas in mixtures containing only azo dyes there is commonly an increase in the absorption band associated with the dye that absorbs at shorter wavelengths. The spectral differences observed in mixtures containing a naphthionic acid derivative such as Congo Red are considerably less than those observed when other direct dyes are used, thus providing another instance in which amino groups do not appear to be as favorable to the interaction as do hydroxyl groups. Stilbene dyes such as Brilliant
Yellow or Chrysophenine $\mathrm{G}$ and the nonsubstantive benzidine dye Acid Anthracene Red 3B give almost additive spectra with these colorless substances (see fig. 5a). This lack of interaction can probably be attributed to interference by the sulfonic acid groups in the molecules.

\subsection{Other Factors and Their Effect on the Interaction of Direct Azo Dyes}

After this general survey on the relationship between the constitution of dyes and their interaction in aqueous solution, the effects of other conditions on the interaction were investigated.

\section{a. Alcohol}

It has been clearly demonstrated that alcohol, like pyridine, inhibits the aggregation of azo dyes [15], as well as that of dyes of other types $[1,16]$. Pyridine ( $25 \%$ in aqueous solution) has also been shown to be useful for obtaining additive spectra with mixtures of dyes that normally show interaction in aqueous solution [6]. In view of the apparent relation between agregation and interaction, it is not surprising that the interaction of direct azo dyes is also reduced by the presence of alcohol. For example, in a solution containing 4 percent of alcohol the spectrum of a mixture of Brilliant Yellow and Benzo Blue $3 \mathrm{~B}$ is nonadditive, but the differences are much less than those observed in water alone. The spectrum of the mixture in 20 percent aqueous alcohol is almost additive, while in 40 percent alcohol no differences can be observed between the sum and mixture curves.

However, in absolute alcohol the spectra of mixtures of direct azo dyes or their diorthotolylguanidine salts again give some evidence of a slight interaction. 


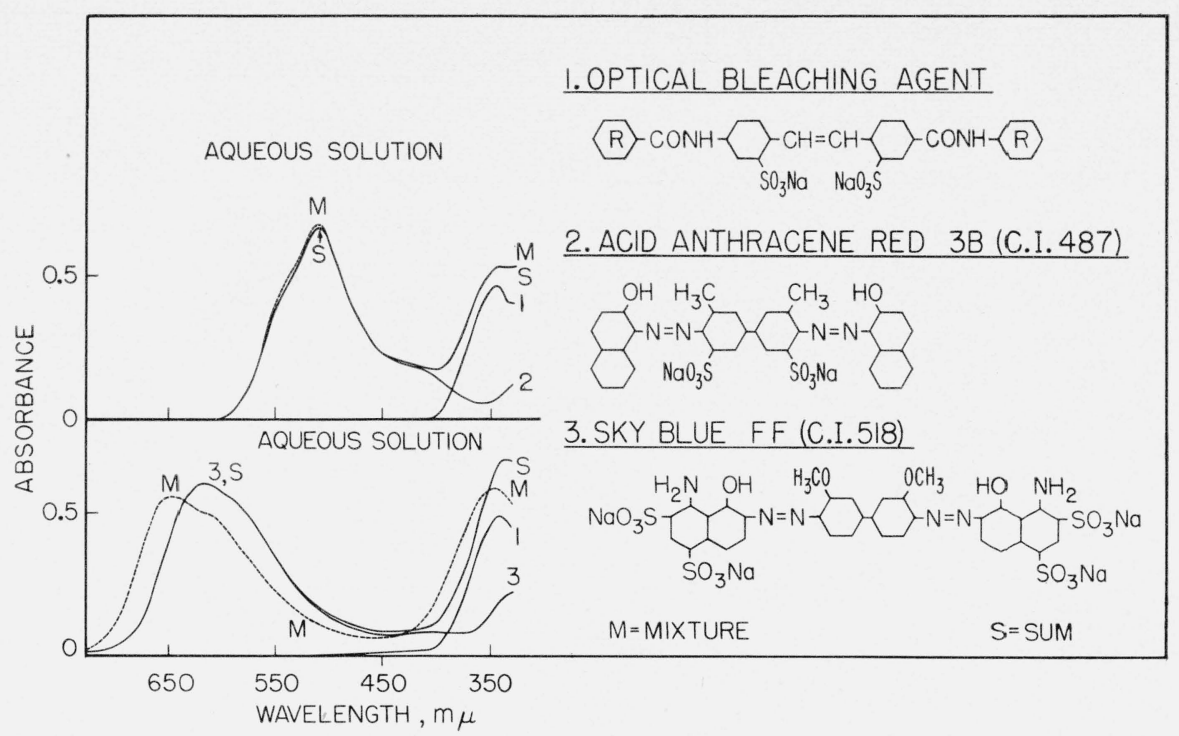

Figure 5. Sum and mixture spectra for an optical bleaching agent (No. 61) (5 mg/liter) with (a) Acid Anthracene Red $3 B$ (16 mg/liter) and with (b) Diamine Sky Blue FF (10 mg/liter).

Length of cells equals $2 \mathrm{~cm}$.

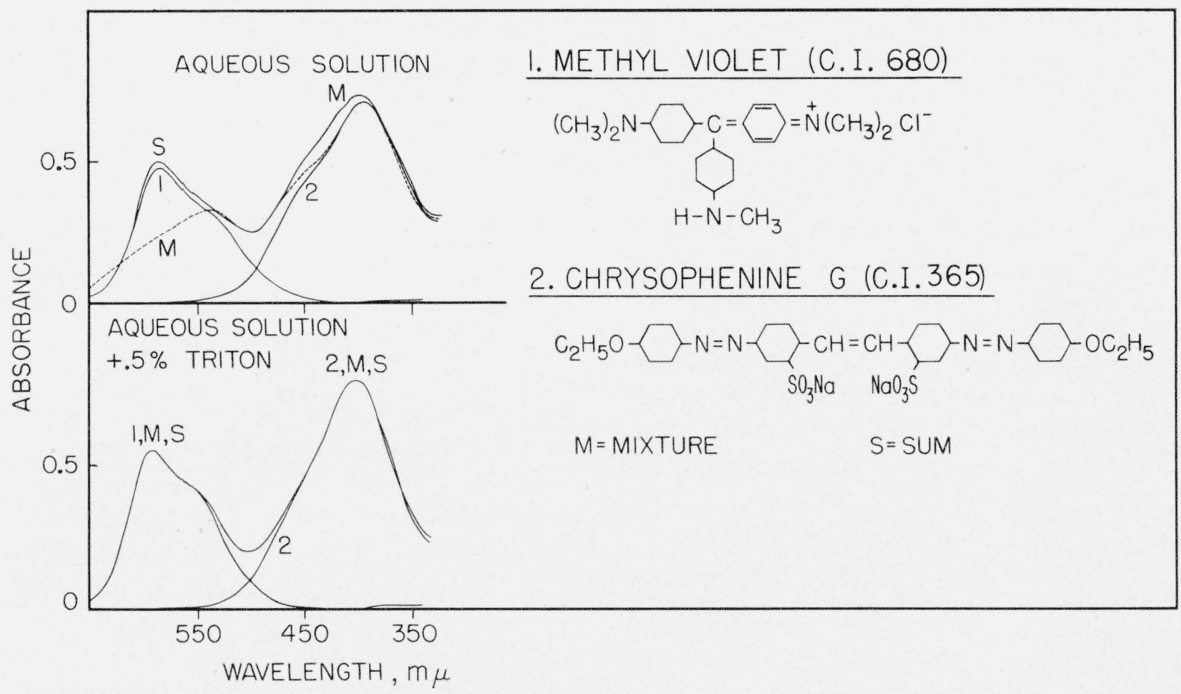

FiguRE 6. Effect of a non-ionic detergent on the sum and mixture spectra for Methyl Violet (2 mg/liter) with Chrysophenine $G$ (7.5 mg/liter $)$.

Length of cells equals $2 \mathrm{~cm}$.

\section{b. Non-Ionic Detergents}

Non-ionic detergents such as polyethylene oxide condensates resemble pyridine, in that they prevent the aggregation of dyes, but are effective at much lower concentrations [17]. Such substances have also been reported [18] to give better results than pyridine when it is desired to obtain additive spectra with mixtures of dyes such as Chrysophenine $\mathrm{G}$ and Diamine Sky Blue FF which normally show interaction. These results are confirmed by the completely additive spectrum observed with a mixture of Brilliant Yellow and Benzo Blue $3 \mathrm{~B}$ in aqueous solution containing 0.1 percent Triton $\mathrm{X}-100$ (a con- densate of ethylene oxide with an octylphenol). A mixture of Chrysophenine $\mathrm{G}$ and Methyl Violet requires slightly more detergent to prevent interaction, for a slight interaction is observed in 0.1 percent Triton X-100 solution, but the spectrum is strictly additive when the concentration of detergent is increased to 0.5 percent (see fig. 6).

The effect of the detergent concentration on these two mixtures is apparently related to the concentration effect that is observed with the individual dyes. At low concentrations Triton $\mathrm{X}-100$ causes changes in the absorption spectra of the individual dyes which are dependent upon the detergent concentra- 
tion. As the amount of detergent is increased, a concentration is reached beyond which no further change in spectrum is observed. With the direct azo dyes this limiting value occurs when the detergent concentration is between 0.03 and 0.05 percent, while the limiting value for Methyl Violet is close to 0.3 percent. In a dye mixture it appears necessary to have the detergent concentration above the limiting concentration for both components of the mixture in order to prevent the interaction of the dyes completely. It is of interest that, while solutions of Triton $\mathrm{X}-100$ contain micelles even at very low concentrations, the monomer saturation concentration (the concentration above which essentially all detergent is in the form of micelles) is 0.3 percent [19].

\section{c. Inorganic Salts}

Since inorganic electrolytes are known to increase the aggregation of many direct dyes, their effect on the interaction of such dyes in mixtures is another factor of interest. Diamine Sky Blue FF (No. 10) and Brilliant Yellow (No. 11) can be taken as representative direct dyes giving nonadditive spectra. Brilliant Yellow is of more value in such studies than the classic example, Chrysophenine G; for it shows no phototropic changes to obscure the spectral effects of interaction, while its structure is similar and its association tendencies in mixtures appear to be identical with those of Chrysophenine G.

Diamine Sky Blue FF is reported to show increasing aggregation with increasing salt concentration [20]. This aggregation is reflected in changes in the absorption spectra (see fig. 7). As the concentration of potassium bromide is increased from 0 to 32 $\mathrm{g}$ /liter, the main absorption band of the dye becomes progressively lower and broader. Although there is no marked change in the position of the maximum, there is an apparent shift in the center of gravity of the band toward longer wavelengths. These changes do not appear to approach a limit at the maximum salt concentration used. Potassium sulfate produces similar changes in the spectrum of the dye. Both salts produce similar changes in the spectra of other related benzidine dyes (e. g., Benzo Azurine G, No. 8; Dianil Blue G, No. 9; and Benzo Blue 3B, No. 6), but with these dyes the maxima are shifted slightly to shorter wavelengths.

Since Brilliant Yellow is structurally similar to Chrysophenine $\mathrm{G}$, which is not aggregated by salts [10], it would also be expected to be unaffected by salts. The spectrum of Brilliant Yellow is found to show no change as the concentration of potassium bromide is increased from 0 to $16 \mathrm{~g}$ /liter, indicating that probably no aggregation occurs.

When mixtures of Diamine Sky Blue FF and Brilliant Yellow are prepared in solutions containing increasing concentrations of potassium bromide or potassium sulfate, the absorption band associated with the Brilliant Yellow increases in intensity and is shifted to longer wavelengths (see fig. 8). As the salt concentration is increased, a limiting concentration is reached, beyond which no further changes are

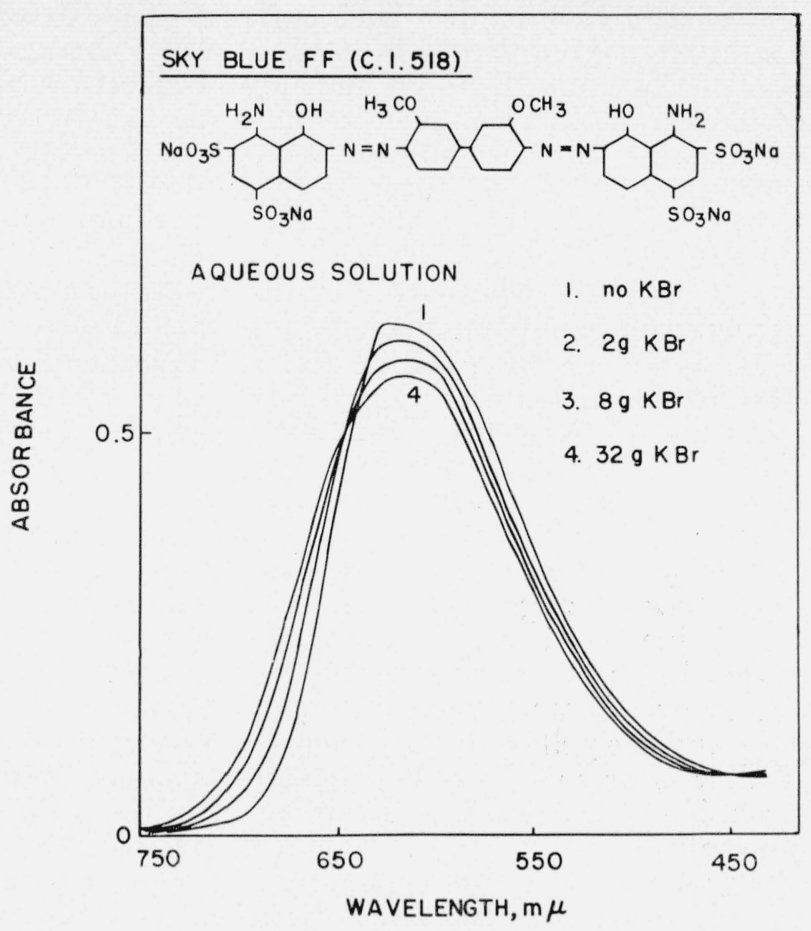

FIGURE 7. Effect of potassium bromide on the absorption spectrum of Diamine Sky Blue FF (10 mg/liter).

Length of cells equals $2 \mathrm{~cm}$.

SKY BLUE FF (C.1.518)

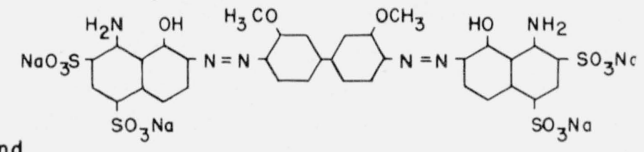

BRILLIANT YELLOW (C. 1.364 )

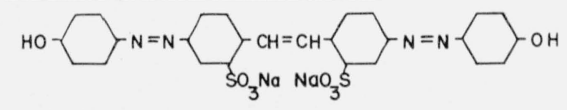

AQUEOUS SOLUTION

I. no $\mathrm{KBr}$

2. $2 \mathrm{~g} \mathrm{KBr}$

3. $8 \mathrm{~g} \mathrm{KBr}$

4. $32 \mathrm{~g} \mathrm{KBr}$

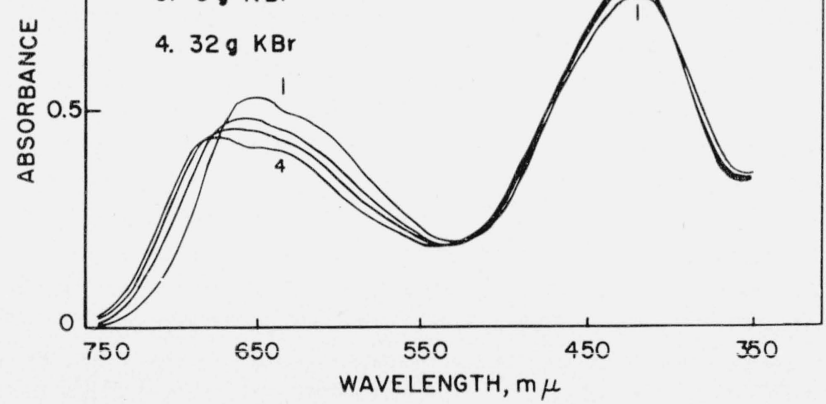

Figure 8. Effect of potassium bromide on the absorption spectrum of a mixture of Diamine Sky Blue FF (10 mg/liter) and Brilliant Yellow (5 $\mathrm{mg} /$ liter $)$.

Length of cells equals $2 \mathrm{~cm}$. 
observed in this band. The spectrum of a mixture containing Diamine Sky Blue FF at a concentration of $10 \mathrm{mg}$ /liter and Brilliant Yellow at a concentration of $5 \mathrm{mg}$ /liter shows no further change in the $400-\mathrm{m} \mu$ region when the potassium bromide concentration is made greater than $4 \mathrm{~g} /$ liter, but $1 \mathrm{~g} /$ liter of the salt is sufficient to produce the maximum change when the concentration of Brilliant Yellow in the mixture is $2.5 \mathrm{mg}$ /liter. The nature of the spectral changes observed in this band suggests that the addition of salts causes an increase in the interaction of the dyes in the mixture.

As the salt concentration is increased, the absorption band associated with the Diamine Sky Blue FF also changes in a manner suggesting increased interaction; that is, the band undergoes a bathochromic shift together with a decrease in intensity. However, these changes continue as the salt concentration is increased and a limit to the changes was not reached at a salt concentration of $32 \mathrm{~g} /$ /iter. Similar results are obtained when different proportions of the blue and the yellow dye are used, even when Brilliant Yellow is present in sufficient excess to make it improbable that any free Diamine Sky Blue FF is present in the mixture. The progressive spectral changes observed with increasing salt concentration in this latter case resemble those produced by the action of salts on Diamine Sky Blue FF in the absence of Brilliant Yellow, and suggest an aggregation of the Brilliant Yellow-Diamine Sky Blue FF complex similar to that of the blue dye alone.

\section{d. Concentration Changes}

Another factor that can cause increased aggregation of dyes is increased concentration. This effect is particularly marked with certain basic dyes [2] but has also been demonstrated in the case of direct azo dyes [4].

A qualitative estimate of the effect of concentration on the spectrum of a dye can be obtained by making a series of measurements with dye solutions in both sample and reference cells. A fixed concentration difference is maintained between the solutions in the two cells while the total concentrations of the dye in both are increased. The spectral changes of Diamine Sky Blue FF observed in this manner, as the concentration in the sample cell is increased from 20 to $100 \mathrm{mg}$ /liter while the concentration difference between the sample and reference cells is fixed at $20 \mathrm{mg} /$ liter, are very similar to those observed with a fixed concentration of dye as the salt concentration is increased from 0 to $5 \mathrm{~g}$ /liter (see fig. 9). The effects of aggregation on the spectrum of the dye are thus similar in the two cases.

In the case of Brilliant Yellow, which is not appreciably aggregated, there is no noticeable change in spectrum as the concentration is increased.

With mixtures of Brilliant Yellow and Sky Blue $\mathrm{FF}$, increasing the concentration of one component brings about changes in the absorption band associated with the other component very similar to the changes produced by increasing the salt concentration. When the concentration of Brilliant Yellow is

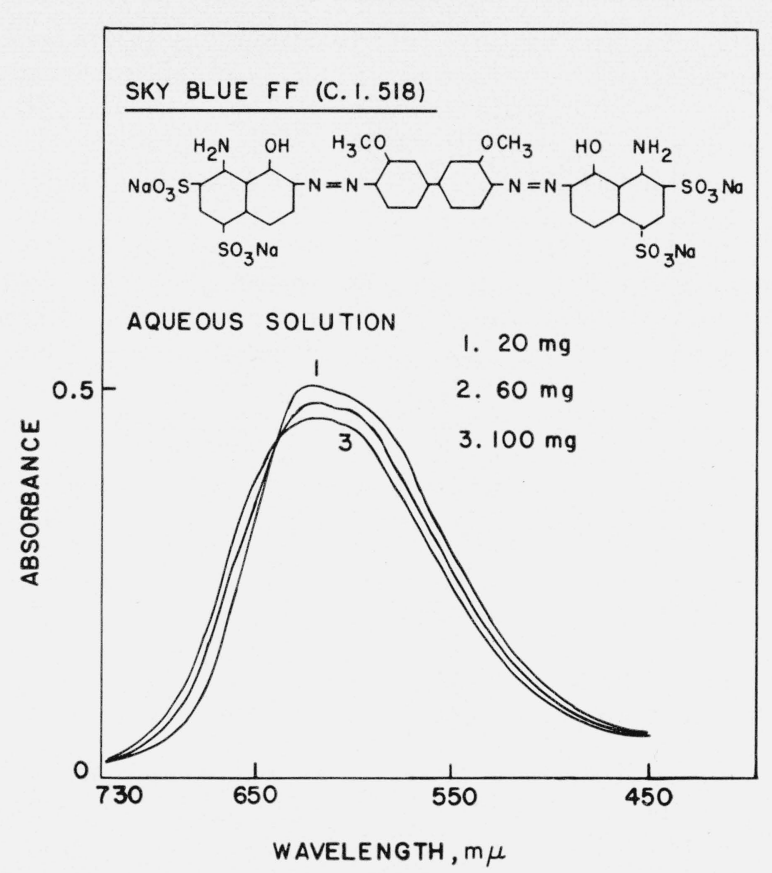

FIGURE 9. Effect of concentration on the absorption spectrum of Diamine Sky Blue FF.

1, Concentration of dye in sample cell equals $20 \mathrm{mg} /$ liter. Reference cell contains water. 2, Concentration of dye in sample cell equals $60 \mathrm{mg} / \mathrm{liter}$; in reference cell equals $40 \mathrm{mg} / \mathrm{liter} .3$, Concentration of dye sample cell equals $100 \mathrm{mg} / \mathrm{liter}$; in reference cell equals $80 \mathrm{mg} /$ liter. Length of cell equals $2 \mathrm{~cm}$.

fixed at $5 \mathrm{mg}$ /liter, its absorption band undergoes progressive bathochromic and hyperchromic shifts as the concentration of Diamine Sky Blue FF is increased from 0 to $20 \mathrm{mg} /$ liter. For concentrations of the blue dye between 20 and $100 \mathrm{mg} /$ liter, no further change is observed in this band.

When the concentration of Diamine Sky Blue FF is fixed at $4 \mathrm{mg} /$ /iter, an increase in the concentration of Brilliant Yellow brings about both a lowering and a bathochromic shift of the absorption band of the blue dye (see fig. 10). The curves obtained with small increments in the concentration of Brilliant Yellow show a single, well-defined isosbestic point until a concentration of $0.75 \mathrm{mg}$ /liter is reached. Such an isosbestic point is to be expected if there is an equilibrium between the ions of the individual dyes and a single form of the Brilliant Yellow. Diamine Sky Blue FF complex, since Brilliant Yellow shows no absorption in this region. However, as the concentration of Brilliant Yellow is increased further, the isosbestic point is no longer observed, while the bathochromic, hypochromic shifts continue. The greatest changes are observed as the concentration of Brilliant Yellow is increased from 0 to 20 $\mathrm{mg} /$ /iter, although concentrations of yellow dye up to $100 \mathrm{mg}$ /liter are effective in producing marked changes, and no limit is observed with concentrations up to $500 \mathrm{mg} /$ liter.

The absence of an isosbestic point, except at the lowest concentrations of Brilliant Yellow, indicates that the mixture contains more than two species absorbing in the region of the main absorption band of the blue dye, and the nature of the spectral changes 


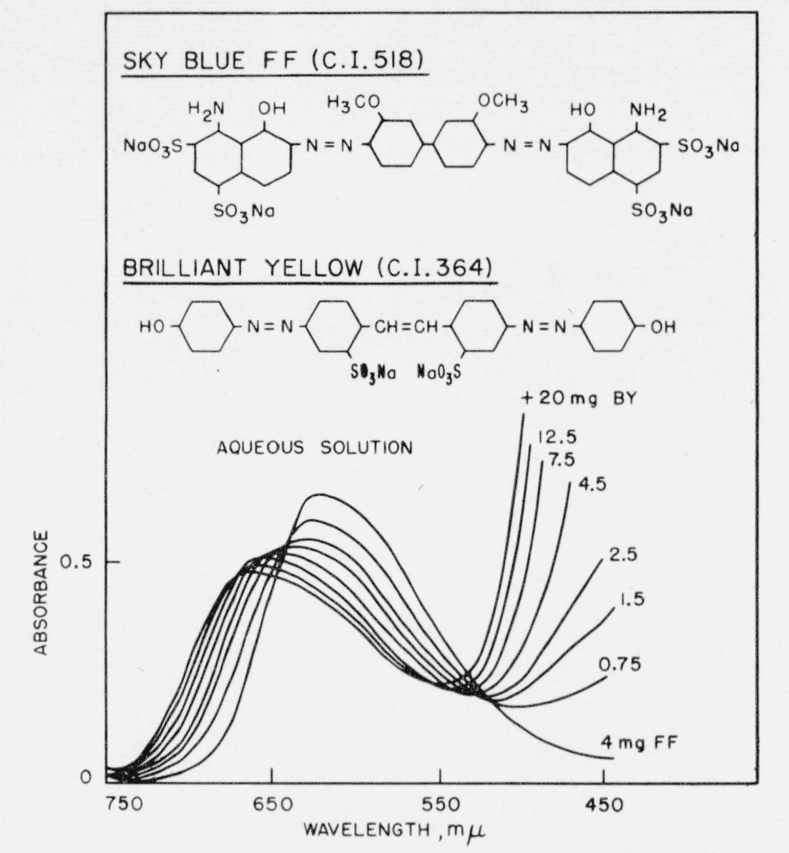

Figure 10. Effect of increasing Brilliant Yellow concentration on the absorption spectrum of Diamine Sky Blue FF.

Length of cell equals $2 \mathrm{~cm}$.

observed suggests that the mixture is quite complex. Similar series of curves, all showing progressive changes in absorption with isosbestic points observed only at the lowest concentrations of the yellow dye and with no indication of limits to the changes at higher concentrations, are also obtained when the concentration of Brilliant Yellow is gradually increased in mixtures containing fixed concentrations of Benzo Azurine G, Dianil Blue G, or Benzo Blue 3 B.

\section{e. Acids and Bases}

In dilute acid solution (0.01 $N$ or below) dyes such as Congo Red (No. 1) change color as a result of the addition of protons. The blue, acid forms of Congo Red and similar dyes give non-additive spectra with Chrysophenine $\mathrm{G}$ and with Brilliant Yellow, thus indicating that the presence of the positively charged groups does not prevent interaction of the dyes. This is to be expected, since the addition of positive charges to the dye molecule should reduce the repulsion by the sulfonic anions and facilitate the interaction of the dyes.

Azo dyes containing hydroxyl groups are not greatly affected by dilute acids, and the spectra obtained with mixtures of such dyes in acid solutions do not differ significantly from nonadditive spectra obtained in distilled water.

In dilute bases, dyes containing hydroxyl groups as the only auxochromes change color with the formation of phenolate ions. In this form such dyes give additive spectra in mixtures where interaction is normally observed in neutral solutions. For example, Brilliant Yellow (No. 11), Benzo Azurine G (No. 8), Dianil Blue G (No. 9), Chrysamine G (No.
3), and Benzo Fast Yellow 5GL (No. 15) give additive spectra with other direct dyes in $0.2 \mathrm{~N}$ sodium hydroxide solutions; while dyes such as Chrysophenine $\mathrm{G}$, Congo Red, or Benzo Blue 3B, which contain either amino groups in addition to hydroxyl groups or else no hydroxyl groups, give nonadditive spectra with other direct dyes in basic solutions as well as in neutral solutions.

In order to obtain interaction of direct dyes, it is necessary that the forces acting to give interaction be large enough to overcome the repulsion of the negative charges localized on the sulfonic acid groups of the dye molecules. Additional negative charges added to the molecule of a hydroxyl-bearing dye by the formation of phenolate ions tend to be distributed over the whole molecule because of the conjugation of the oxygen with the rest of the molecule. The additive spectra obtained in basic solutions of such dyes suggest that the added repulsion resulting from the increased negative charges on the dye molecule is sufficient to prevent interaction with other dyes.

\section{f. Phototropism and Dye Interaction}

It was mentioned earlier that the phototropism of Chrysophenine $\mathrm{G}$ may give rise to misleading results in the study of the spectra of dye mixtures unless suitable precautions are taken. In some cases the phototropic property of this dye can be used to advantage in studying dye interaction.

A mixture of Chrysophenine $\mathrm{G}$ and the nonphototropic dye Diamine Sky Blue FF gives a nonadditive spectrum in aqueous solution by virtue of complex formation. When this mixture is irradiated with blue light, the spectral changes in the $400-\mathrm{m} \mu$ region (the region of maximum absorption for Chrysophenine $\mathrm{G}$ ) resemble those observed with Chrysophenine $\mathrm{G}$ alone (see fig. 11a). The spectrum in the $600-\mathrm{m} \mu$ region (the region where Chrysophenine $\mathrm{G}$ shows no absorption but the absorption of Diamine Sky Blue FF is at a maximum) is also observed to change, although to a much smaller extent. The spectral changes produced in this region by illumination are of such a nature that they suggest a tendency toward less interaction of the two dyes on irradiation [21].

The effect of light on the spectrum of such mixtures leads to the conclusions that the complex itself is not phototropic and that only the more stable, "dark" form of Chrysophenine G is involved in complex formation. Irradiation of the mixture, particularly with blue light, leads to the formation of the less stable forms of Chrysophenine $\mathrm{G}$ with a resultant decrease in the concentration of the dark form. This then shifts the equilibrium between the free dyes and the complex, resulting in a decrease in the concentration of the complex and producing the observed spectral changes. This interpretation of the changes in spectrum is in agreement with that of Atherton and Peters [22].

If these conclusions are correct, it is evident that the effects of irradiation observed in the $600-\mathrm{m} . \mu$ region should represent the extent to which the dye complex is broken up, while the changes in the 400- 


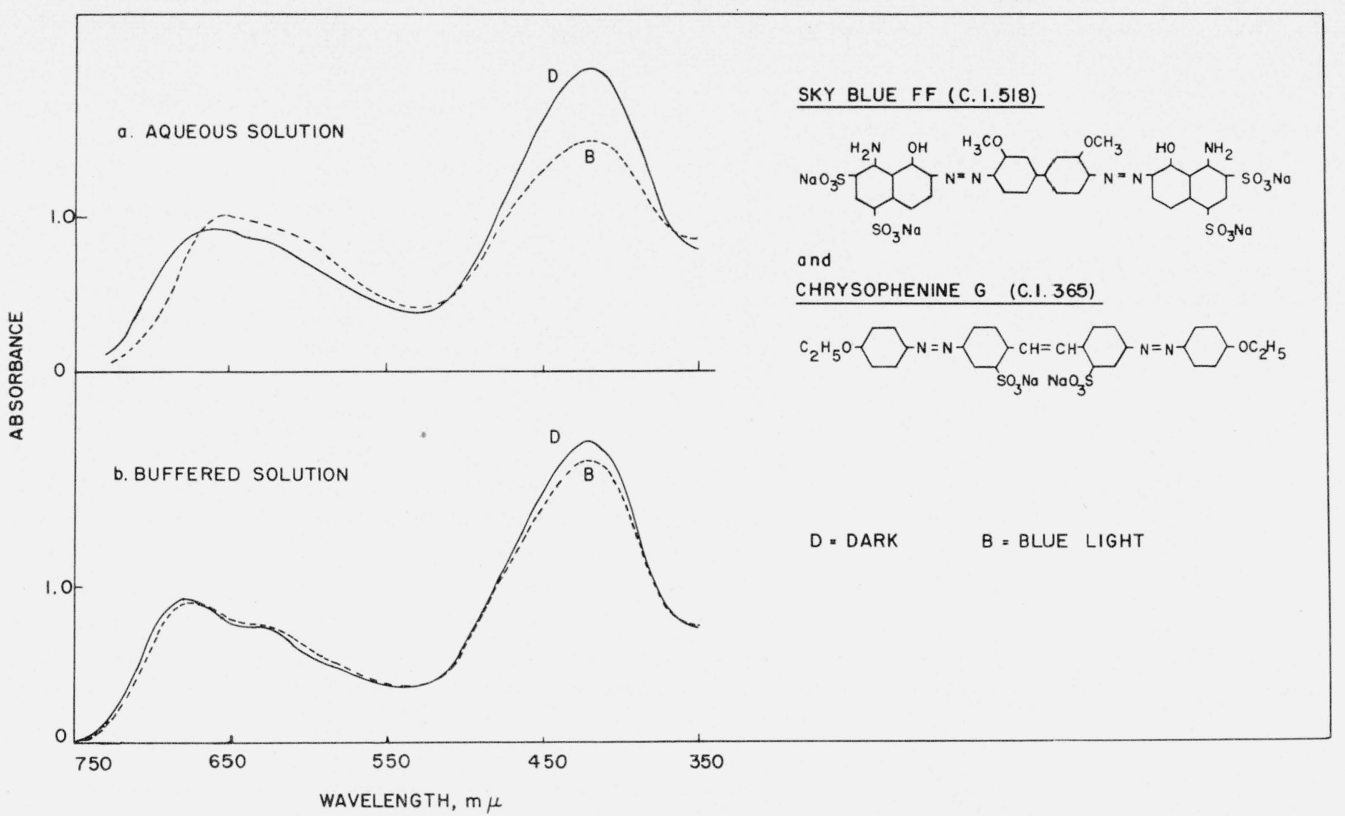

FIGURE 11. Phototropic effect shown by a mixture of Diamine Sky Blue FF (20 mg/liter) and Chrysophenine G (10 mg/liter) in water and in a buffer solution ( $p \mathrm{H}$ equals 5.7).

Length of cell equals $2 \mathrm{~cm}$.

$\mathrm{m} \mu$ region should be related to the concentration of free Chrysophenine $\mathrm{G}$ in the mixture before irradiation, together with that released by the disruption of the complex. Unfortunately, beating of the mixture during irradiation also leads to dissociation of the complex, making the interpretation of the spectral effects more difficult and thus preventing a quantitative study of the effect at this time. In general, the changes observed are those which would be expected if an equilibrium exists between a complex, the dark form of Chrysophenine G, and free Diamine Sky Blue FF. An increase in the concentration of either dye or the addition of salts (or the use of an acetate buffer) results in increased interaction of the dyes, as shown by the relative decrease in the phototropic changes (see fig. 11b).

\section{Conclusions}

The results obtained in this general survey tend to confirm the idea that there is a fundamental relationship between the factors involved in dyeing cotton, in the aggregation of azo dyes, and in the interaction of azo dyes in mixtures. This relationship probably extends to dyes of other types as well.

The exact nature of the forces operative in the dyeing of cotton is still undetermined, as can be seen from the different opinions expressed in the symposium on the physical chemistry of dyeing [23]. It has been found empirically that several features in the structure of direct azo dyes tend to increase the substantivity of the dye, although the existence of substantive dyes that do not possess some of these features shows that many of these are not absolute requirements for substantivity and suggests that several types of interaction are responsible for the binding of dyes to cotton. Some of the features mentioned [24] as required or desirable for substantivity are linearity of the dye molecule, coplanarity of the molecule, the presence of hydrogenbonding groups, the presence of a sufficiently long chain of conjugated double bonds, and the presence of a minimum number of solubilizing groups, the latter preferably arranged along one side of the molecule with hydrogen-bonding groups on the other side. The results of this survey indicate that these features also tend to favor the interaction of dyes in mixtures. Solubilizing groups such as the sulfonic acid group appear to be more effective in preventing interaction or the aggregation of individual dyes than in decreasing substantivity; this is apparent in the case of Chrysophenine G, which is substantive although the sulfonic acid groups prevent its aggregation, or its interaction with other dyes bearing suitably placed sulfonic acid groups.

One apparent contradiction can be noted. The presence of amino groups increases the substantivity of dyes, particularly on viscose [25], while the results given in this paper suggest a tendency for dyes containing amino groups as the only auxochromes to show less interaction in mixtures. Griffiths and Neale point out that the greater substantivity on viscose may result from the larger number of carboxyl groups on viscose than on cotton. If such is the case, the opposite effects of the amino group on the substantivity and on the interaction of dyes would not be surprising.

Other parallels may be drawn between dyeing, aggregation, and interaction. Increased dye concentration and increased salt concentration have been shown to cause increased aggregation of direct azo dyes as well as increased interaction in mixtures; 
these changes also increase the rate and depth of dyeing of cotton. Similarly, stripping agents such as pyridine which remove dyes from fibers prevent the aggregation or the interaction of these dyes.

Probably the most significant similarity between these three phenomena is seen in the spectral changes they produce. The results given in this paper illustrate changes in spectrum brought about by aggregation due to increased salt concentration or increased dye concentration, as well as those resulting from the interaction of dyes. A comparison of these changes with those resulting from the dyeing of cellulose sheet by a number of substantive benzidine dyes [26] shows marked similarities, particularly in the direction of the spectral shifts as well as in the increased asymmetry on the long wavelength side of the absorption band.

Since work on this paper was completed and tentatively reported at the symposium of the Chemical Society of London in February 1956 [27], a paper by Derbyshire and Peters on the interaction between Chlorazol Sky Blue FF and Chrysophenine G has appeared [28]. From a study of the spectral changes caused by variations in the relative concentrations of the two dyes in aqueous solution, they conclude that a 1:1 complex is formed, provided that the Chrysophenine $\mathrm{G}$ is not in excess, and that complexes richer in the yellow dye are formed when higher concentrations of Chrysophenine G are used. Our results with Brilliant Yellow are consistent with the formation of such a 1:1 complex at low concentrations of yellow dye. However, the lack of a second isosbestic point as the concentration of the yellow dye is increased and the similarity between the effect of inorganic salts and the effect of increasing dye concentration on the spectrum of the mixture suggest a salting-out process rather than the formation of complexes richer in the yellow dye. From the heat of complex formation and from a study of the spectra of these dyes and dye mixtures on Cellophane, Derbyshire and Peters provide more evidence that the mechanism of complex formation is similar to that of dye adsorption by fibers.

Washington, August 5, 1957

\section{References}

[1] E. Rabinowitch and L. F. Epstein, J. Am. Chem, 'Soc. 63, 69 (1941).

[2] S. E. Sheppard and A. L. Geddes, J. Am. Chem. Soc. 66, 1995, 2003 (1944).

[3] L. Michaelis, J. Phys. Colloid Chem. 54, 1 (1950).

[4] For a review of the literature, see H. A. Standing, Trans. Faraday Soc. 41, 410 (1945).

[5] G. Scheibe, Kolloid Z. 82, 1 (1938).

[6] S. M. Neale and W. A. Stringfellow, J. Soc. Dyers Colourists 59, 241 (1943).

[7] D. R. Lemin and T. Vickerstaff, Trans. Faraday Soc. 43, 491 (1947).

[8] W. R. Brode, J. H. Gould, and G. M. Wyman, J. Am. Chem. Soc. 74, 4641 (1952).

[9] K. Ventkataraman, The chemistry of synthetic dyes, p. 499, 1288 (Academic Press, Inc., New York, N. Y., 1952).

[10] F. H. Holmes and H. A. Standing, Trans. Faraday Soc. 41, 542, 568 (1945).

[11] T. Vickerstaff, The physical chemistry of dyeing, p. 187 (Interscience Publishers, New York, N. Y., 1950).

[12] E. Valko, J. Soc. Dyers Colourists 55, 174 (1939); K. N. Davies and J. Whetstone, J. Chem. Soc., 1954, 865; A. N. Derbyshire, Trans. Faraday Soc. 51,909 (1955).

[13] K. Ventkataraman, op. cit., [9] p. 277.

[14] D. W. Hein and E. S. Pierce, J. Am. Chem. Soc. 76 2725 (1954).

[15] J. T. Martin and H. A. Standing, J. Text. Inst. 40, T671 (1949).

[16] S. E. Sheppard, Rev. Modern Phys. 14, 303 (1940).

[17] J. T. Martin and H. A. Standing, J. Text. Inst. 40, T689 (1949).

[18] H. H. Taylor and F. T. Simon, Am. Dyestuff Reptr. 34, $319(1945)$

[19] L. M. Kushner and W. D. Hubbard, J. Phys. Chem. 58, 1163 (1954).

[20] E. Valko, Trans. Faraday Soc. 31, 230 (1935).

[21] W. R. Brode in The Roger Adams symposium, p. 50 (John Wiley \& Sons, Inc., New York, N. Y., 1955).

[22] E. Atherton and R. H. Peters, Recent advances in the chemistry of colouring matters, Special Publication No. 4, London: The Chemical Society, p. 26 (1956).

[23] The physical chemistry of dyeing and tanning. Discussions Faraday Sos., No. 16 (1954).

[24] T. Vickerstaff, The physical chemistry of dyeing, 2d ed., p. 183 (Interscience Publishers, New York, N. Y., 1950).

[25] L. H. Griffiths and S. M. Neale, Trans. Faraday Soc. 30, 3955 (1934).

[26] D. Krüger and H. Rudow, Ber. y1, 707 (1938).

[27] W. R. Brode, Recent advances in the chemistry of colouring matters, Special Publication No. 4, London: The Chemical Society, p. 1 (1956).

[28] A. N. Derbyshire and R. H. Peters, J. Soc. Dyers Colourists, 72, 268 (1956). 